\title{
A Retrospective Evaluation of Remote Pharmacist Interventions in a Telepharmacy Service Model Using a Conceptual Framework
}

Jayashri Sankaranarayanan, MPharm, PhD, 1,2

Lori J. Murante, PharmD, ${ }^{3}$ and Lisa M. Moffett, PharmD ${ }^{4}$

${ }^{1}$ School of Pharmacy, Department of Pharmacy Practice, University of Connecticut, Hartford Hospital, Hartford, Connecticut.

${ }^{2}$ College of Pharmacy, Department of Pharmacy Practice, University of Nebraska Medical Center, Omaha, Nebraska.

${ }^{3}$ Alternate Site Pharmacies \& Clinical Decision Support,

The Nebraska Medical Center, Omaha, Nebraska.

${ }^{4}$ Remote Pharmaceutical Care, Drug Information Service, The Nebraska Medical Center, Omaha, Nebraska.

\begin{abstract}
Objectives: This retrospective cross-sectional study evaluated a telepharmacy service model using a conceptual framework to compare documented remote pharmacist interventions by year, hospital, and remote pharmacist and across rural hospitals with or without an onsite rural hospital pharmacist. Materials and Methods: Documented remote pharmacist interventions for patients at eight rural hospitals in the Midwestern United States during prospective prescription order review/entry from 2008 to 2011 were extracted from RxFusion ${ }^{\circledR}$ database (a home-grown system, i.e., internally developed program at The Nebraska Medical Center (TNMC) for capturing remote pharmacistdocumented intervention data). The study authors conceptualized an analytical framework, mapping the 37 classes of remote pharmacist interventions to three broader-level definitions: (a) intervention, eight categories (interaction/potential interaction, contraindication, adverse effects, anticoagulation monitoring, drug product selection, drug regimen, summary, and recommendation), (b) patient medication management, two categories (therapy review and action), and (c) health system-centered medication use process, four categories (prescribing, transcribing and documenting, administering, and monitoring). Frequencies of intervention levels were compared by year, hospital, remote pharmacist, and hospital pharmacy status (with a remote pharmacist and on-site pharmacist or with a remote pharmacist only) using chisquared test and univariate logistic regression analyses, as appropriate. Results: For 450,000 prescription orders 19,222 remote pharmacist interventions were documented. Frequency of interventions significantly increased each year (36\% in 2009, 55\% in 2010, and 7\% in $2011)$ versus the baseline year $(2008,3 \%)$ when service started. The frequency of interventions also differed significantly across the eight hospitals and 16 remote pharmacists for the three defined intervention levels and categories. Remote pharmacist interventions at hospitals
\end{abstract}

with an on-site and remote pharmacist $(\mathrm{n}=12,141)$ versus those with a remote pharmacist alone $(\mathrm{n}=7,081)$ were significantly more likely to be (1) patient-centered, (2) related to "actionable" medication management recommendations (unadjusted odds ratio $[O R]=1.12$ ), and (3) related to the "transcribing" $(O R=1.47)$ and "prescribing" $(O R=1.40)$ steps of the health system-centered medication use process level (all $\mathrm{p}<0.01)$. Conclusions: This is one of the first studies to demonstrate the patient- and health system-centered nature of pharmaceutical care delivered via a telepharmacy service model by evaluating documented remote pharmacist interventions with an analytical framework.

Key words: pharmacy, telemedicine, medication records, e-health, policy

\section{Background and Significance}

W hen pharmacists are not available in rural communities and when rural hospitals lack resources to staff a pharmacy 24 h/day, 7 days/week, quality and safety of medication use may be compromised., ${ }^{1,2}$ Telepharmacy, which is a subset of telemedicine, may be useful for improving pharmacy service coverage regardless of where the pharmacist is located. ${ }^{2-4}$ Telepharmacy applies to activities including a combination of electronic data entry, prescription order verification, centralized anticoagulation clinics, online benefit adjudication, medication dispensing, and telehealth consultation with medication use evaluation using computer technology. ${ }^{1,2}$ Several rural hospitals in the United States ${ }^{4}$ and Australia ${ }^{5}$ have implemented telepharmacy services that allow for 24-h pharmacist review of all medication orders. In the United States, rural hospitals with or without on-site hospital pharmacists were incentivized by regulatory mandates from the Centers for Medicare and Medicaid Services and other regulatory agencies ${ }^{6}$ to use computers and health information technology (HIT) as a vehicle for providing convenient and affordable pharmacy service solutions to meet patient needs. By implementing telepharmacy services, critical access hospitals with 25 or fewer beds in rural areas could qualify to receive incentive payments from the 2009 Medicare and Medicaid electronic health record (EHR) incentive programs for eligible professionals and eligible hospitals that adopt, implement, upgrade, or demonstrate "meaningful use" of certified EHR technology. ${ }^{7}$

\section{THE NEBRASKA MEDICAL CENTER TELEPHARMACY SERVICE}

Frequently, telepharmacy services administered via "excess capacity hub and spoke" models ${ }^{8}$ generally utilize pharmacists in urban 


\section{SANKARANARAYANAN ET AL.}

hospitals to triage and enter medication orders for rural hospital partners. In 2008, The Nebraska Medical Center (TNMC) in Omaha implemented a remote pharmacy model that tailors the service (excluding remote retail dispensing) to fit the needs of the rural hospital site. ${ }^{9}$ The current TNMC model employs 16 part-time home-based pharmacists to provide remote pharmacy services for inpatients at rural hospitals. These remote pharmacists in TNMC service model (appropriately licensed for each state) work from home using secure virtual private network connections to the rural hospital sites.

TNMC and each rural hospital site enter into a business associate agreement along with a 2-year master service agreement. Each site tailors the service to their needs and technology, choosing from a menu of services offered: prospective medication order review, entry, or verification in the patient's electronic medical record, visual authentication of medications prior to administration or other use of videoconferencing, policy development for medication use, medication safety or clinical consultations. ${ }^{8}$ Secure virtual private network tunnels are used for connectivity (Fig. 1). Secure credentials are issued by each site for all pharmacists. Each rural hospital establishes a methodology for backup access during connectivity interruptions.
Superuser liaisons use a Web-based tool to record training sessions that can be accessed on demand when needed by remote pharmacists. A shared Web site on TNMC intranet includes "tip sheets" for commonly encountered questions, and details about each site are used for quick reference.

\section{STUDY RATIONALE AND SIGNIFICANCE}

There is very limited knowledge evaluating the place of documented remote pharmacist interventions in a telepharmacy service model across the quality of care continuum and with or without an on-site pharmacist located in rural hospitals. Our study addresses important gaps in this knowledge. First, we developed a framework to categorize remote pharmacist interventions provided at three levels across the care continuum: intervention, patient, and health system (throughout the medication use process from transcribing to administration). This framework will enhance the design of an electronic data capture system that will help train current and future pharmacists to improve and coordinate medication therapy management for patients remotely. Second, we describe trends in the intervention categories by year, hospital, and remote pharmacist within the telepharmacy service model. Third, to understand place

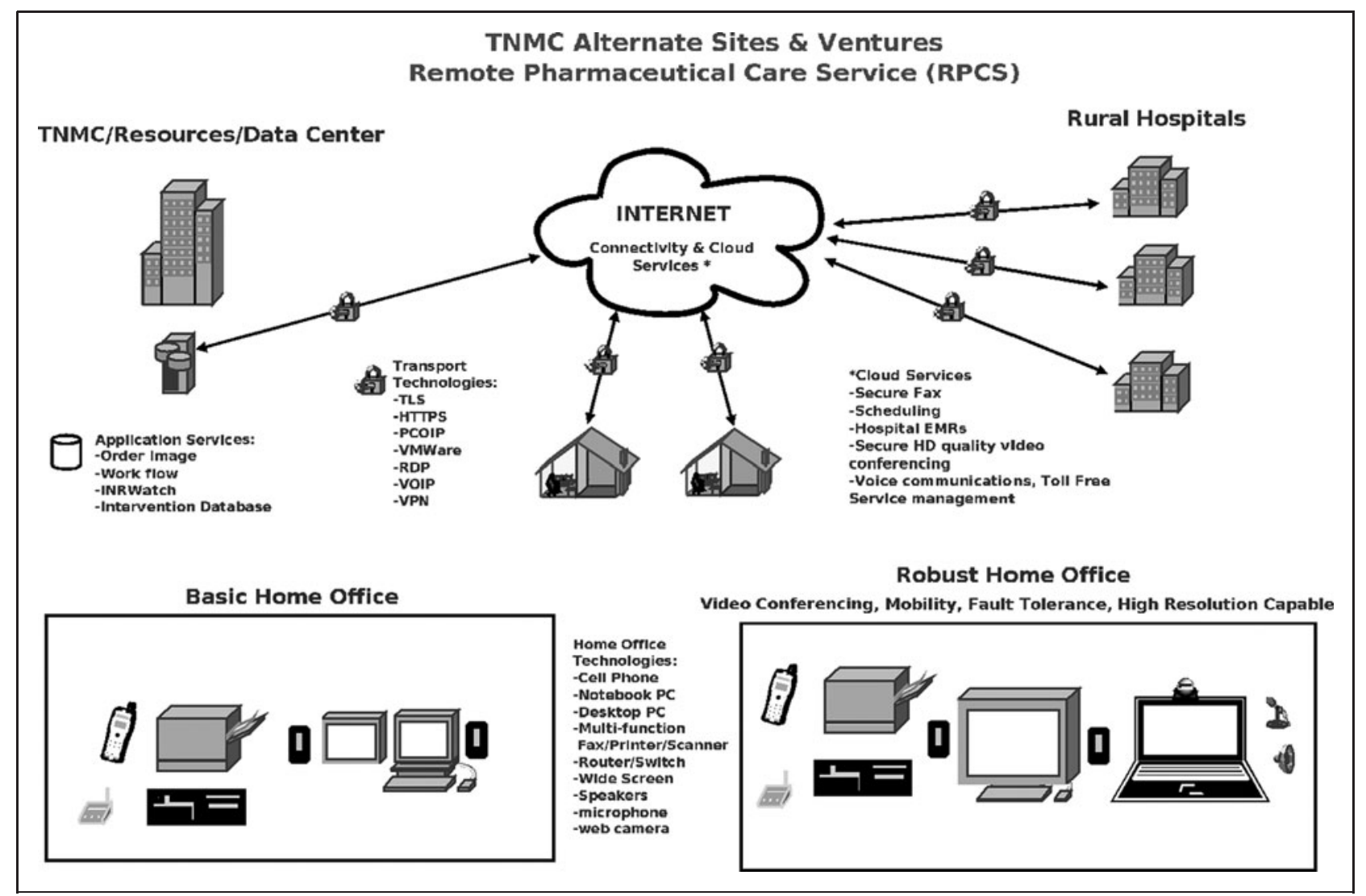

Fig. 1. Structure of The Nebraska Medical Center's (TNMC's) telepharmacy service model. ${ }^{9}$ EMR, electronic medical record; HD, highdefinition; TLS, transport layer security; HTTPS, hypertext transfer protocol secure; PCOIP, PC-over-IP (PCoIP) technology powers virtual workplace; VMWare, virtualization and cloud computing software; RDP, remote desktop protocol; VOIP, voice over Internet protocol; VPN, virtual private network. 
of the remote pharmacist along with the on-site rural hospital pharmacist, we compared intervention categories across hospitals served by a remote pharmacist with and without an on-site hospital pharmacist.

\section{OBJECTIVES}

This retrospective cross-sectional study evaluated a telepharmacy service model using a conceptual framework with three definitions at the intervention level, the patient-centered medication management level, and the health-system centered medication use process level to compare documented remote pharmacist interventions by year, hospital, and remote pharmacist and across rural hospitals with and without an on-site rural hospital pharmacist.

\section{Materials and Methods}

We conducted a retrospective study using descriptive and analytic methods. A software application (OrderImage; Health Care Systems Inc., Montgomery, AL) was used to establish the number of prescription orders processed during the period June 2008-November 2011. Data for the study were extracted from the RxFusion ${ }^{\circledR}$ clinical interventions database (a home-grown system, i.e., internally developed program at TNMC for capturing remote pharmacist-documented intervention data) where the remote pharmacists documented all interventions at the eight hospitals (six critical access hospitals in very rural areas of Nebraska and Iowa, one community hospital each in Nebraska and in a moderately rural area in eastern Iowa). These hospitals were receiving the telepharmacy services during the study time period for prospective order review and/or entry. Details of the eight hospitals (number of licensed beds [bed size], average daily census, telepharmacy service start dates, and onsite pharmacist presence) were collected (Table 1). Anonymized information was reported for the interventions without unique protected health information elements of patients. The authors'

\begin{tabular}{|c|c|c|c|c|}
\hline HOSPITAL $^{a}$ & $\begin{array}{l}\text { SERVICE } \\
\text { START }\end{array}$ & $\begin{array}{l}\text { APPROXIMATE DAILY AVERAGE } \\
\text { NUMBER OF PATIENTS }\end{array}$ & $\begin{array}{c}\text { ON-SITE } \\
\text { PHARMACIST } \\
\text { PRESENT }^{\mathrm{b}}\end{array}$ & $\begin{array}{l}\text { HOSPITAL } \\
\text { LOCATION }\end{array}$ \\
\hline 1 & August 2008 & 10 & No & Nebraska \\
\hline 2 & October 2008 & 12 & Yes & Nebraska \\
\hline 3 & January 2009 & $6-8$ & Yes & Nebraska \\
\hline 4 & February 2009 & $10-12$ & No & Nebraska \\
\hline 5 & October 2009 & $12-14$ & Yes & Nebraska \\
\hline 6 & November 2009 & $6-8$ & Yes & lowa \\
\hline 7 & March 2010 & $10-12$ & No & Nebraska \\
\hline 8 & October 2010 & 25 & Yes & lowa \\
\hline
\end{tabular}

${ }^{\mathrm{a} A l l}$ have 25 or fewer beds.

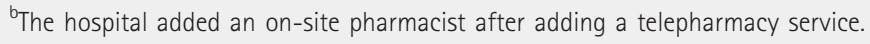

institutional review board approved the study as exempt from human subject requirements.

In TNMC telepharmacy service, medication orders transmitted from the rural hospital to a fax server are viewed by remote pharmacists in an OrderImage application. When the remote pharmacists interact (via a secure Web browser) with the rural hospital's information technology system to provide pharmacy services, they access and review the patient's electronic medical record using their clinical expertise and document relevant pharmacy interventions (e.g., wrong drug, dose, route, interval, etc.) in the RxFusion clinical interventions database. In practice, safety-related or urgent pharmaceutical care interventions were delivered verbally over the phone by the remote pharmacists to the rural hospital providers, and potentially routine or nonurgent interventions were delivered via a written recommendation faxed to the provider at the rural hospital for his or her consideration. These faxed remote pharmacist interventions are placed in the patient's paper medical record or placed as a clinical note in the patient's electronic health record for providers to consider during patient rounds. All the documented remote pharmacist interventions in the RxFusion database were the unit of analyses for this study.

\section{CONCEPTUAL FRAMEWORK TO MAP REMOTE PHARMACEUTICAL CARE INTERVENTIONS}

The study authors (the outcomes researcher, J.S.; the pharmacist administrator and director of the remote pharmacy service program, L.J.M.; and the remote clinical pharmacist, L.M.M.) reviewed the 37 classes of remote pharmaceutical care interventions (Table 2). Then, based on the literature on medication therapy management ${ }^{10,11}$ and using the steps in the medication use process proposed by United States Pharmacopoeia, ${ }^{12}$ the study authors conceptualized an analytical framework, mapping the 37 classes of remote pharmacist interventions to three broader-level definitions: (a) intervention, eight categories (interaction/potential interaction, contraindication, adverse effects, anticoagulation monitoring, drug product selection, drug regimen, summary, and recommendation), (b) medication management, two categories (medication therapy review and medication action), and (c) health system medication use process, four categories (prescribing, transcribing and documenting, administering, and monitoring) (Fig. 2).

\section{STATISTICAL ANALYSES}

To describe trends in the provision of telepharmacy services, the median total number of documented interventions by half the number of hospitals and remote pharmacists across hospitals and pharmacists, respectively, was estimated. To investigate differences in frequencies of 


\begin{tabular}{|c|c|c|}
\hline NUMBER & $\begin{array}{l}\text { INTERVENTION } \\
\text { ENTRY }\end{array}$ & DETAILS \\
\hline 1 & Admission & $\begin{array}{l}\text { Medical condition required additional } \\
\text { or new medication }\end{array}$ \\
\hline 2 & Admission & $\begin{array}{l}\text { Order entry/printing medication } \\
\text { administration record/communication }\end{array}$ \\
\hline 3 & Admission & Preventive therapy ordered \\
\hline 4 & $\begin{array}{l}\text { Adverse drug } \\
\text { reaction-reporting }\end{array}$ & Reporting adverse drug reactions \\
\hline 5 & $\begin{array}{l}\text { Adverse drug } \\
\text { reaction-treatment }\end{array}$ & $\begin{array}{l}\text { Treatment for an adverse drug } \\
\text { reaction }\end{array}$ \\
\hline 6 & Drug allergy & $\begin{array}{l}\text { Review patient's drug allergies before } \\
\text { medications }\end{array}$ \\
\hline 7 & $\begin{array}{l}\text { Alternative route } \\
\text { recommendation }\end{array}$ & Dosage form \\
\hline 8 & Clarification of order & Dose/route/schedule/start/stop time \\
\hline 9 & $\begin{array}{l}\text { Anticoagulation } \\
\text { monitoring }\end{array}$ & Therapy/INR/PT/dose/signs/symptoms \\
\hline 10 & $\begin{array}{l}\text { Drug compatibility } \\
\text { information }\end{array}$ & $\begin{array}{l}\text { Provide drug compatibility } \\
\text { information }\end{array}$ \\
\hline 11 & Pharmacist consultation & $\begin{array}{l}\text { Medical condition required additional } \\
\text { or new drug }\end{array}$ \\
\hline 12 & Pharmacist consultation & Preventive therapy ordered \\
\hline 13 & Pharmacist consultation & $\begin{array}{l}\text { Provide therapeutic information to } \\
\text { prescriber }\end{array}$ \\
\hline 14 & Contraindication & $\begin{array}{l}\text { Drug/diagnosis/age/condition/ } \\
\text { laboratory values/allergy }\end{array}$ \\
\hline 15 & $\mathrm{CrCl}$ & Calculation of $\mathrm{CrCl}$ \\
\hline 16 & $\begin{array}{l}\text { Dose and/or regimen } \\
\text { adjustment }\end{array}$ & Dose therapy duration too long \\
\hline 17 & $\begin{array}{l}\text { Dose and/or regimen } \\
\text { adjustment }\end{array}$ & Dose therapy duration too short \\
\hline 18 & $\begin{array}{l}\text { Dose and/or regimen } \\
\text { adjustment }\end{array}$ & Dose too high \\
\hline 19 & $\begin{array}{l}\text { Dose and/or regimen } \\
\text { adjustment }\end{array}$ & Dose too low \\
\hline 20 & $\begin{array}{l}\text { Dose and/or regimen } \\
\text { adjustment }\end{array}$ & Dose adjustment \\
\hline 21 & Drip rate calculation & For nurses \\
\hline 22 & Drug information & $\begin{array}{l}\text { Includes literature search/ } \\
\text { manufacturer information }\end{array}$ \\
\hline 23 & $\begin{array}{l}\text { Duplicate/unnecessary } \\
\text { therapy }\end{array}$ & Duplicate/unnecessary therapy \\
\hline 24 & Formulary substitution & Formulary substitution \\
\hline
\end{tabular}

\begin{tabular}{|c|c|c|}
\hline NUMBER & $\begin{array}{c}\text { INTERVENTION } \\
\text { ENTRY }\end{array}$ & DETAILS \\
\hline 25 & $\begin{array}{l}\text { Home medication rec- } \\
\text { onciliation }\end{array}$ & $\begin{array}{l}\text { Medicaiton review/outpatient } \\
\text { pharmacy consultation }\end{array}$ \\
\hline 26 & Medication identification & $\begin{array}{l}\text { Nurses calling the pharmacist about } \\
\text { patient's medication identification } \\
\text { based on color, shape, and marking } \\
\text { on the medication }\end{array}$ \\
\hline 27 & Incomplete order & Incomplete order \\
\hline 28 & Drug interaction & $\begin{array}{l}\text { Drug/drug or drug/food or } \\
\text { drug/diagnosis }\end{array}$ \\
\hline 29 & Pharmacokinetic consult & Initial dose and follow-up \\
\hline 30 & Medication error & Medication error reporting \\
\hline 31 & Monitoring lab values & BMP/CBC/drug levels/SCr/BUN \\
\hline 32 & Patient profile review & $\begin{array}{l}\text { Medical condition requires additional/ } \\
\text { new medications }\end{array}$ \\
\hline 33 & Patient profile review & Preventive therapy ordered \\
\hline 34 & Patient profile review & $\begin{array}{l}\text { Medication review verifying that all } \\
\text { administration times are appropriate } \\
\text { and any administration precautions } \\
\text { are so noted on electronic medical } \\
\text { record for nursing }\end{array}$ \\
\hline 35 & $\begin{array}{l}\text { Therapeutic recommen- } \\
\text { dation form }\end{array}$ & Completed/faxed to remote site \\
\hline 36 & $\begin{array}{l}\text { Shift transfer } \\
\text { communication }\end{array}$ & Between remote pharmacists \\
\hline 37 & TPN & TPN calculations \\
\hline
\end{tabular}

BMP, basic metabolic panel; BUN, blood urea nitrogen; $C B C$, complete blood count; $\mathrm{CrCl}$, creatinine clearance; INR, international normalized ratio; PT, prothrombin time; $\mathrm{SCr}$, serum creatinine; TPN, total parenteral nutrition.

interventions over time across years, across the eight hospitals, and across the 16 remote pharmacists, chi-squared or Fisher's exact tests were used as appropriate. A Bonferroni correction was applied for more than two group comparisons. To investigate the association of each of the three definition levels of remote intervention categories with remote pharmacist coverage but with or without an on-site hospital pharmacist (dependent variable of interest), univariate logistic regression analyses was used. All analyses were carried out using a standard statistical analysis software (SAS version 9.3; SAS Institute, Cary, NC).

\section{Results}

About half a million prescription orders $(n=450,000)$ were processed for the eight study hospitals over the 2.5-year study period (Table 3). In total, 19,222 remote pharmacist interventions documented in the RxFusion database were evaluated. Compared 
Level 1. Intervention

8 categories: Recommendation, Drug Product Selection, Summary, Drug Regimen, Adverse Effects, Anticoagulation Monitoring, Potential Interaction or Interaction, Contraindication

Level 2. Patient-centered medication management

2 categories: Medication Therapy Review, Medication Action

Level 3. Health system-centered medication use process* 4 categories: Prescribing, Transcribing, Administering, Monitoring

* Note that because remote pharmacists in a telepharmacy service do not dispense medications to patients at hospitals but could document interventions related to administering medications, the dispensing step category was excluded and administering category was retained.

Fig. 2. Levels and categories for mapping pharmaceutical care interventions from Table 2. with 520 interventions in base year 2008, the number of interventions increased every year (6,832 interventions in 2009, 10,481 interventions in 2010, and 1,389 interventions in 2011). On average, 2,403 interventions (range, 846-5,307) were delivered per hospital. More than 1,807 (median) total interventions were documented by each of the four (out of the eight total) hospitals. On average, 1,201 interventions (range, 190-4,183) were documented by each of the 16 pharmacists over the 2.5-year period. More than 743 (median) total interventions were documented by each of the eight (out of the 16 total) remote pharmacists. Overall, the frequency of interventions differed significantly across the 4 years ( $p<0.01$; Table 3$)$, across the eight hospitals ( $p<0.01$; data not shown), and across the 16 remote pharmacists ( $p<0.05$; data not shown) for the eight proposed intervention level categories, two patientcentered medication management level categories, and four health system-centered medication use process level categories, respectively.

Compared with hospitals with a remote pharmacist alone (i.e., without an on-site pharmacist), hospitals with both an

\begin{tabular}{|c|c|c|c|c|c|c|}
\hline DEFINITION LEVEL AND CATEGORIES & FY2008 & FY2009 & FY2010 & FY2011 & TOTAL (\%) & PVALUE $^{\mathrm{a}}$ \\
\hline Total $(\%)$ & $5,209(3 \%)$ & $6,832(36 \%)$ & $10,481(55 \%)$ & $1,389(7 \%)$ & $19,222(100 \%)$ & \\
\hline Intervention & & & & & & $<0.0006$ \\
\hline Recommendation & 217 & 3,078 & 4,647 & 652 & $8,594(45 \%)$ & \\
\hline Drug regimen & 73 & 245 & 241 & 49 & $608(3 \%)$ & \\
\hline Adverse effects & 11 & 185 & 331 & 37 & $564(3 \%)$ & \\
\hline Anticoagulation monitoring & 24 & 106 & 202 & 88 & $420(2 \%)$ & \\
\hline Potential interaction or interaction & 8 & 54 & 52 & 11 & $125(1 \%)$ & \\
\hline Medication action & 320 & 4,788 & 6,878 & 901 & $12,887(67 \%)$ & \\
\hline Medication therapy review & 200 & 2,044 & 3,603 & 488 & $6,335(33 \%)$ & \\
\hline Health system-centered medication use process & & & & & & $<0.0006$ \\
\hline Prescribing & 224 & 4,050 & 6,296 & 945 & $11,515(60 \%)$ & \\
\hline Transcribing & 180 & 2,391 & 3,689 & 434 & $6,694(35 \%)$ & \\
\hline Administering & 8 & 64 & 130 & 6 & $208(1 \%)$ & \\
\hline Monitoring & 108 & 327 & 366 & 4 & 805 (4\%) & \\
\hline
\end{tabular}

annivariate associations of the three intervention levels each across the 4 years were studied using chi-squared tests for differences of association between the categorical variables. All $p$ values less than 0.05 were significant. A Bonferroni correction was applied for more than 2 group comparisons. 


\section{SANKARANARAYANAN ET AL.}

Table 4. Frequency of Remote Pharmaceutical Care Interventions at Hospitals With or Without an On-Site Pharmacist Along with Remote Pharmacist Coverage, 2008-2011 $(n=19,222)$

HOSPITAL WITH NO ON-SITE

PHARMACIST BUT WITH REMOTE

DEFINITION LEVEL AND CATEGORIES PHARMACIST $(N=7,081)$

HOSPITAL WITH ON-SITE

PHARMACIST AND REMOTE PHARMACIST $(N=12,141)$

TOTAL PVALUE ${ }^{\mathrm{a}}$

Intervention

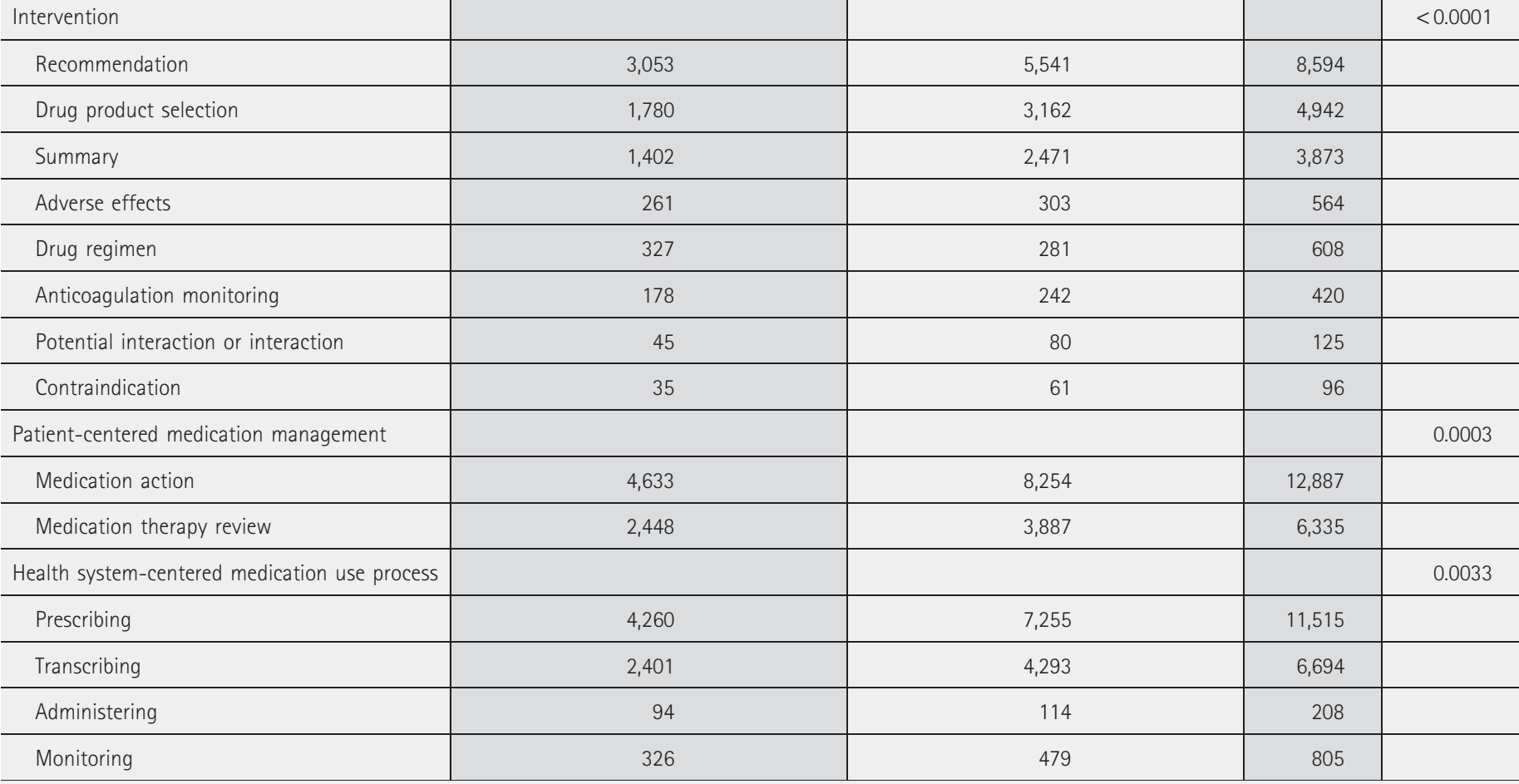

anivariate association of each of the three intervention definition levels across the two groups of hospital pharmacy status were studied using chi-squared tests for differences of association between the categorical variables. All $p$ values less than 0.05 were significant. A Bonferroni correction was applied for more than 2 group comparisons.

on-site and remote pharmacist had a significantly higher frequency of interventions related to recommendations, drug product selection, summary, and drug regimen, followed by adverse effects, anticoagulation monitoring, potential interactions, and contraindications (Table 4). They also showed a higher frequency of interventions related to taking actions regarding medication use rather than interventions related to review of medications and a higher frequency of interventions related to prescribing and transcribing, followed by monitoring and administering functions.

There were significant differences between hospitals with both an on-site and remote pharmacist $(n=12,141)$ versus with remote pharmacist coverage alone (i.e., without an on-site pharmacist) $(n=7,081)$ for the three category levels (Table 5). Recommendation (odds ratio $[\mathrm{OR}]=1.34 ; 95 \%$ confidence interval $[\mathrm{CI}], 1.10-1.63$; $p \leq 0.0001)$, drug product selection (OR $=1.31 ; 95 \% \mathrm{CI}, 1.07-1.60$; $p=0.0002)$, and summary (OR $=1.30 ; 95 \% \mathrm{CI}, 1.06-1.59 ; p=0.0007)$ interventions each versus anticoagulation monitoring were significantly more likely to be associated with hospitals with both an on-site and remote pharmacist versus hospitals with remote pharmacist coverage alone (i.e., without an on-site pharmacist). However, drug regimen $(\mathrm{OR}=0.63 ; 95 \% \mathrm{CI}, 0.49-0.81 ; p \leq 0.0001)$ versus anticoagulation monitoring intervention was significantly less likely to be associated with hospitals with both an on-site and remote pharmacist versus hospitals with remote pharmacist coverage alone (i.e., without an on-site pharmacist). Interventions related to taking actions regarding medication use (OR $=1.12 ; 95 \% \mathrm{CI}, 1.05-1.19 ; p=0.0003)$ versus interventions related to review of medications were significantly more likely to be associated with hospitals with both an on-site and remote pharmacist versus hospitals with remote pharmacist coverage alone (i.e., without an on-site pharmacist). Transcribing $(\mathrm{OR}=1.47 ; 95 \% \quad \mathrm{CI}, 1.12-1.95 ; p=0.0003)-$ and prescribing $(\mathrm{OR}=1.07 ; 95 \% \mathrm{CI}, 1.07-1.85 ; p=0.0095)$-related interventions each versus administering related interventions were significantly more likely to be associated with hospitals with both an on-site and remote pharmacist versus hospitals with remote pharmacist coverage alone (i.e., without an on-site pharmacist). However, monitoring- versus administering-related interventions were not significantly different across hospitals with both an on-site and remote pharmacist and hospitals with remote pharmacist coverage alone (i.e., without an onsite pharmacist) ( $p=0.5565)$. 


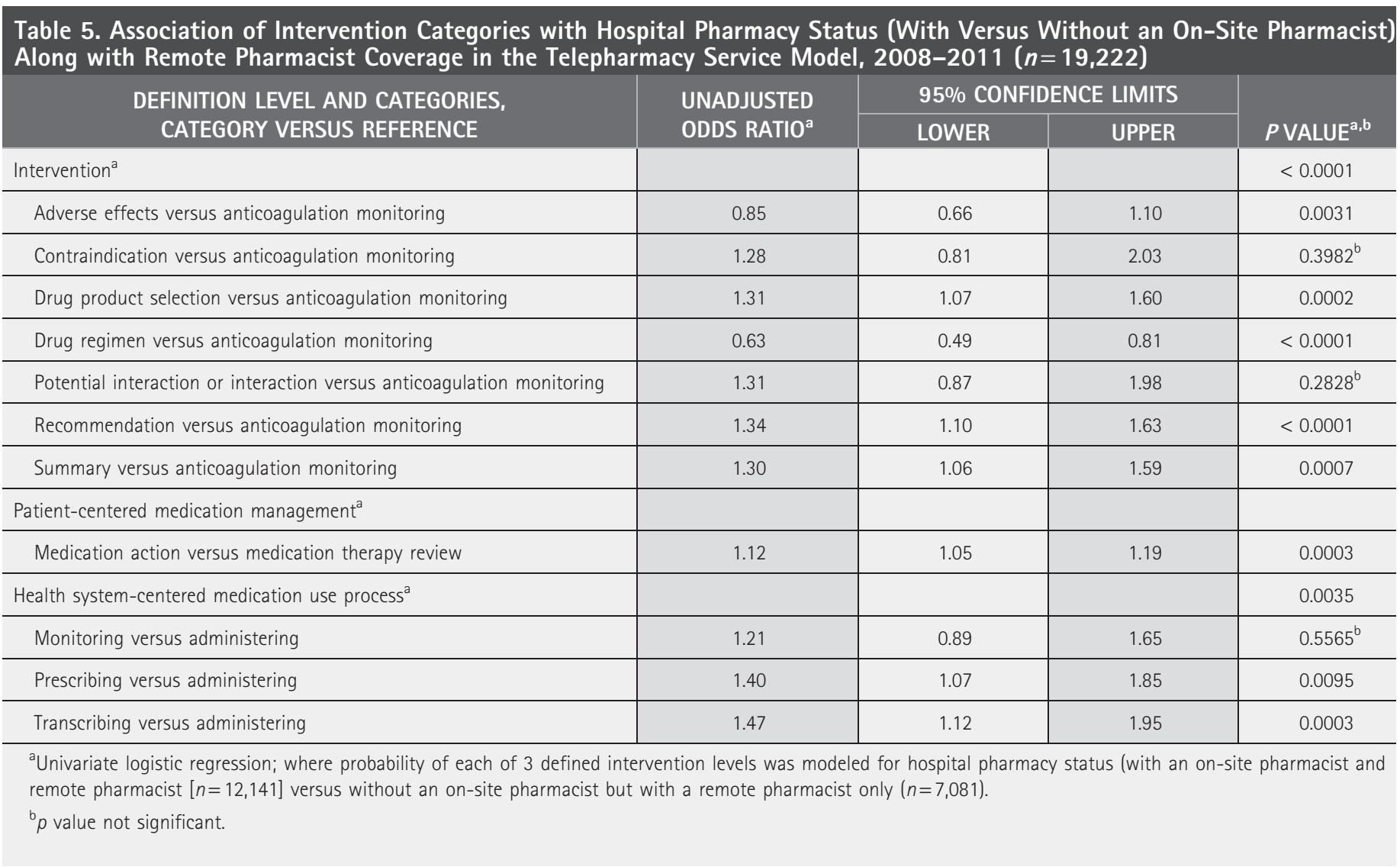

\section{Discussion}

To our knowledge the present study is one of the first to focus on remote pharmaceutical care interventions within a telepharmacy service model delivered to rural hospitals. We developed an analytical framework to evaluate the telepharmacy service model with three definitions at the intervention level, the patient-centered medication management level, and the health system-centered medication use process level and also compared the trends in these remote pharmacist interventions by year, hospital, and remote pharmacist and across hospitals with and without an on-site hospital pharmacist.

Rural hospitals in several states in the United States (for example, Washington State, ${ }^{13}$ the hospital telepharmacy network in North Dakota, ${ }^{14-16}$ Colorado, ${ }^{17}$ Alaska, ${ }^{18}$ Iowa, ${ }^{8,19}$ Kansas, ${ }^{20,21}$ Florida, ${ }^{22}$ and Maryland ${ }^{23}$ ) and outside the United States (Australia ${ }^{5}$ ) now use telepharmacy services. Previous studies have focused on describing telepharmacy activities in a small number of hospitals ${ }^{5,8,13-21}$ or in a single hospital. $^{22,23}$ Studies have reported the impact of telepharmacy by tracking medication error rates, ${ }^{4}$ by describing pharmaceutical care activities focused on drug-related problems, ${ }^{5}$ by evaluating number of orders reviewed, modified, discontinued, and voided by remote pharmacists, ${ }^{9}$ by describing the 24 -h pharmacy staffing coverage given, ${ }^{15}$ or by showing increased speed of prescription order processing, ${ }^{19,22}$ re- ducing order processing time, freeing up pharmacists for quality-enhancing initiatives, or saving money. ${ }^{21}$

We found that the number of categories of proposed interventions, patient-centered interventions, and health system-centered interventions significantly increased from the baseline first year of implementation of the telepharmacy service. The proposed interventions, patient-centered interventions, and health system-centered interventions were also significantly different among the eight hospitals in the study as well as among the 16 remote pharmacists who provided and documented the pharmaceutical care interventions. We also found that hospitals with both an on-site and remote pharmacist versus hospitals with a remote pharmacist alone (i.e., without an on-site pharmacist) were significantly more likely to have remote pharmaceutical care interventions (1) that were patientcentered rather than drug-centered, (2) that had actionable recommendations rather than medication review related to medication use at the patient-centered level, and (3) that were related to the transcribing order and prescribing steps rather than administering step of the medication use process at the health system-centered level. These study findings suggest that remote pharmacists complement the onsite rural hospital pharmacist in their work and enhance the patientcenteredness in delivery of pharmaceutical care.

The present study demonstrates the potential for remote pharmacist interventions within a telepharmacy service model to 


\section{SANKARANARAYANAN ET AL.}

coordinate patient-centered pharmaceutical care and to also facilitate the meaningful use of EHRs. ${ }^{6,7}$ This supports the guidance from a Pharmacy e-Health Information Technology Collaborative white paper calling for integrating pharmacy HIT into the national HIT/EHR infrastructure for safe and effective medication use. ${ }^{24}$ The current types of evaluations could help telepharmacy service providers and rural hospitals demonstrate the "meaningful use" of certified EHR technology and get qualified for getting incentive payments from the Medicare EHR incentive program. ${ }^{7}$ Therefore, the current study findings would be of interest to payers, providers, and researchers in developing quality metrics for evaluations to guide reimbursement coverage for telepharmacy services beyond the "meaningful use" of EHRs. This is important given the growing acceptance of telemedicine by providers, policy makers, consumers, and insurers to increase access, improve quality, and reduce costs in the delivery of healthcare. In this context, the American Telemedicine Association's State Policy Toolkit has reported all 50 state Medicaids and 18 private insurers have or are adopting mandates for coverage of some aspects of telemedicine services. ${ }^{25}$ Thus, continued demonstration projects studying the place and value of remote pharmaceutical care interventions and how they are facilitating the process of safe medication use could also help pharmacists become eligible professionals to qualify for these Medicare EHR incentive payments; however, all these are areas for future exploration.

\section{LIMITATIONS}

Several limitations could have affected the study estimates. First, because several hospitals were contracted for the remote pharmacy service at different dates, the trends across different hospitals for the calculated frequencies and types of remote pharmaceutical care interventions at the three definition levels would have been affected. Second, lack of time may have made pharmacists only record important interventions so that the study results may be an underestimate. This is because, like in all service delivery models, it is difficult to allow appropriate time to document every single intervention that a remote pharmacist does during the course of his or her duties or changes to the service provision. Of note is that although our study was limited to 2008-2011 data, following our evaluation, the telepharmacy service has grown to serve 13 remote sites. The remote pharmacists experienced an increase in workload due to an increase in order volume. Furthermore, some sites wanted the remote pharmacists to document interventions specifically in the patient's medical records within their pharmacy computer system. Thus, in 2011, for the sake of avoiding the duplicative burden of documenting interventions, remote pharmacists were instructed to only document interventions that significantly impacted patient safety and therapy. Consequently, a trend of documenting fewer interventions in the current home-based RxFusion documentation system emerged in the year 2011, which is also reflected in the lower number of remote interventions documented in 2011 in the current study. Furthermore, there were differences in documentation of remote care interventions from one pharmacist to the other, which suggests that technical and motivational training and reimbursement of these services are impor- tant to consider in future studies. Third, the effect of missing information in a patient's medical records on documented interventions and corroboration of these effects were beyond the scope of this study's objectives. Fourth, as in other studies, personal bias could also have affected study estimates because the same remote pharmacist delivered and documented the interventions. Fifth, our study was based entirely on interventions documented by the remote pharmacists. Although all documented interventions are considered to be implemented at hospitals, we were unable to corroborate whether all the interventions recorded by the remote pharmacists as recommendations were accepted and implemented by pharmacists and other healthcare professionals on-site at the hospitals as this was beyond the scope of the study.

Finally, data collection and assessment of the appropriateness or clinical impact, effectiveness, cost-effectiveness, and the delivery channels (phone, fax, etc.) of the remote pharmacist interventions were beyond the scope of this study. However, these limitations are the basis for future research and can potentially be built into the design of the electronic data capture system.

The delivery of pharmaceutical care services across the continuum of care in rural settings will call for the optimization of pharmacist services and the ability to capture meaningful data in the EHR. Numerous barriers continue to limit the ability to capture all pharmaceutical care value. Therefore, future research needs to address several areas: for example, (1) study opportunities to enhance the design of an efficient electronic data capture system that would enable remote pharmacists to document interventions with ease and would motivate all remote pharmacists to equally document their interventions, (2) use this framework to continuously evaluate in real-time the place and value of remote pharmaceutical care interventions in the current telepharmacy model and other models, and (3) use the framework developed to elucidate the growing role of pharmacists in medication therapy management within a telepharmacy model when patients transition from inpatient to outpatient settings of care.

In the long term, our study results will provide a framework that providers, administrators, and payers can adapt in the evaluation processes of telepharmacy service models. This will help stakeholders understand the significance of remote pharmaceutical care interventions to elucidate the critical role of the remote pharmacist in improving the quality of medication use processes within the current and other similar telepharmacy service delivery models.

\section{Conclusions}

This is one of the first studies to demonstrate to providers, payers, and policymakers about the patient- and health system-centered nature of pharmaceutical care delivered via a telepharmacy service model by evaluating documented remote pharmacist interventions with an analytical framework. With further validation and considering areas of future research, this framework could enhance the design of electronic data capture of documented interventions and can be used as a quality metric tool for routine real-time evaluations of telepharmacy service models. In summary, such studies and tools can enhance the quality of pharmaceutical care in rural as well as urban healthcare systems that will be of interest to providers, payers, and policymakers. 


\section{EVALUATION OF A TELEPHARMACY SERVICE DELIVERY MODEL}

\section{Acknowledgments}

The authors thank the remote pharmacists who documented the interventions they made in the telepharmacy service model. We also thank Richard VanCura (associated with The Nebraska Medical Center) who prepared the data for the study and Lynette Smith, MS (biostatistician in the College of Public Health at the University of Nebraska Medical Center) who assisted with analysis.

\section{Disclosure Statement}

No competing financial interests exist.

\section{Author Contributions}

J.S. led the conceptualization, design, data collection planning, statistical analyses, interpretation of data, and drafting of the manuscript. L.J.M. was involved in the conceptualization, design, data collection planning, data acquisition, interpretation of data, and critical revision of manuscript. L.M.M. was involved in the conceptualization, design, data collection, reviewing, and critical revision of manuscript. All authors gave final approval.

\section{REFERENCES}

1. Angaran DM. Telemedicine and telepharmacy: Current status and future implications. Am J Health Syst Pharm 1999;56:1405-1426.

2. MacDowell M, Glasser $M$, Fitts $M$, Nielsen $K$, Hunsaker M. A national view of rural health workforce issues in the USA. Rural Remote Health 2010;10:1531.

3. Focus group on telepharmacy. Am J Health Syst Pharm 2001;58:167-169.

4. Casey MM, Sorensen TD, Elias W, Knudson A, Gregg W. Current practices and state regulations regarding telepharmacy in rural hospitals. Am J Health Syst Pharm 2010;67:1085-1092.

5. Poulson LK, Nissen L, Coombes I. Pharmaceutical review using telemedicine-A before and after feasibility study. J Telemed Telecare 2010;16:95-99.

6. Centers for Medicare and Medicaid Services. A record of progress on health information technology. April 2013. Available at: www.cms.gov/Newsroom/ MediaReleaseDatabase/Fact-Sheets/2013-Fact-Sheets-Items/2013-04-23.html (last accessed October 25, 2013).

7. Centers for Medicare and Medicaid Services. EHR incentive programs. Available at: www.cms.gov/Regulations-and-Guidance/Legislation/

EHRIncentivePrograms/index.html?redirect=/ehrincentiveprograms/ (last accessed October 25, 2013).

8. Wakefield DS, Ward MM, Loes JL, O'Brien J, Sperry L. Implementation of a telepharmacy service to provide round-the-clock medication order review by pharmacists. Am J Health Syst Pharm 2010;67:2052-2057.

9. The Nebraska Medical Center. Remote pharmaceutical care. Available at: www.nebraskamed.com/pharmacy/remote-pharmaceutical-care (last accessed October 25, 2013).

10. Joint Initiative of the American Pharmacists Association and the National Association of Chain Drug Stores Foundation. Medication therapy management in pharmacy practice core elements of an MTM service model version 2.0. March 2008. Available at: www.pharmacist.com/sites/default/ files/files/core_elements_of_an_mtm_practice.pdf (last accessed October 25, 2013).

11. Moczygemba LR, Barner JC, Gabrillo ER, Godley PJ. Development and implementation of a telephone medication therapy management program for Medicare beneficiaries. Am J Health Syst Pharm 2008;65:1655-1660.

12. USP medication use process model. Available at: www.achca.org/content/ pdf/LTCPLC_Stmt3_MedUseProcess_081031.pdf (last accessed October 25, 2013).
13. Lordan D, Vorhees N, Richards C. Telepharmacy offers hope for rural hospitals. National pharmacist shortage prompts innovative approach to hospital-based services in Washington State. Telemed Today 2002;9:13-15.

14. Peterson CD, Anderson HC Jr. The North Dakota Telepharmacy Project: Restoring and retaining pharmacy services in rural communities. J Pharm Technol 2004;20:28-39.

15. Young D. Telepharmacy project aids North Dakota's rural communities. Am J Health Syst Pharm 2006;63:1776, 1779-1780.

16. Peterson CD, Scott DM, Rathke A, Killingsworth P, Hill G. Establishing a central order entry system for delivering telepharmacy services to remote rural hospitals. J Pharm Technol 2010;26:179-186.

17. Colorado Rural Health Center. Rural telepharmacy services. December 2008. Available at: www.coruralhealth.org/crhc/cah/cah_news/CO\%20Rural \%20Telepharmacy\%20Services\%20Report_12\%208\%2008.pdf (last accessed October 25, 2013).

18. Rose JL. Improved and expanded pharmacy care in rural Alaska through telepharmacy and alternative methods demonstration project. Int J Circumpolar Health 2007;66(Suppl 1):14-22.

19. Boon AD. Telepharmacy at a critical access hospital. Am J Health Syst Pharm 2007;64:242-244.

20. Garrelts JC, Gagnon M, Eisenberg C, Moerer J, Carrithers J. Impact of telepharmacy in a multihospital health system. Am J Health Syst Pharm 2010;67:1456-1462.

21. One 14-hospital telepharmacy program in Kansas that includes one rural hospital in its program has been reported to reduce order processing time, free up pharmacists for quality-enhancing initiatives, and saves money. Available at: www.innovations.ahrq.gov/evidencerating.aspx (last accessed October 25, 2013).

22. Woodall SC. Remote order entry and video verification: Reducing after-hours medication errors in a rural hospital. Jt Comm J Qual Saf 2004;30:442-447.

23. Keeys CA, Dandurand K, Harris J, Gbadamosi L, Vincent J, Jackson-Tyger B, King J. Providing nighttime pharmaceutical services through telepharmacy. Am J Health Syst Pharm 2002;59:716-721.

24. The Pharmacy e-HIT Collaborative 2010: The roadmap for pharmacy health information technology integration in U.S. health care. Available at: www.pharmacyhit.org/index.php/links/webinar-the-roadmap-for-pharmacyhealth-information-technology-integration-in-us-health-care (last accessed October 25, 2013).

25. American Telemedicine Association. State policy toolkit improving access to covered services for telemedicine. Available at: www.americantelemed.org/docs/ default-source/policy/ata-state-policy-toolkit.pdf?sfvrsn $=6$ (last accessed October 25, 2013).

Address correspondence to: Jayashri Sankaranarayanan, MPharm, PhD

School of Pharmacy

Department of Pharmacy Practice University of Connecticut Hartford Hospital Hartford, CT 06102-5037

E-mail: jsankara@live.com jsankara@unmc.edu jsankara@uconn.edu

Received: December 9, 2013

Revised: January 10, 2014

Accepted: January 10, 2014 